\title{
Avaliação microbiológica de ostras cultivadas no litoral de Alagoas submetidas a depuração em sistema fechado de recirculação
}

\section{Microbiological evaluation of grown on the coast of Alagoas State submitted to purification in a closed recirculation system}

\author{
Thiago Dias Trombeta*1 \& Ana Cristina Lima Normande ${ }^{2}$ \\ ${ }^{1}$ Departamento de Acuicultura, Universidad Católica del Norte. \\ ${ }^{2}$ Departamento de Nutrição, Universidade Federal de Alagoas - UFAL. \\ *E-mail: thiago_trombeta@yahoo.com.br
}

Recebido: 31 de março de 2017 / Aceito: 22 de setembro de 2017 / Publicado: 8 de dezembro de 2017

\begin{abstract}
Resumo As ostras são moluscos bivalves, filtradores, que podem reter, em seus tecidos, microrganismos patogênicos e causar problemas de saúde publica aos consumidores. Dessa forma, a depuração é um processo que visa eliminar os agentes patogênicos de moluscos bivalves, fornecendo um produto com garantia sanitária ao mercado consumidor. Este trabalho teve como objetivo, realizar uma avaliação microbiológica de ostras cultivadas no litoral de Alagoas e comparar os resultados com os padrões microbiológicos exibidos no Brasil, conforme a resolução da Anvisa RDC n ${ }^{\circ} 12$, de 02 de janeiro de 2001. As ostras foram provenientes de quatro locais de produção, submetidas a quatro tempos de depuração $(12,24,36$ e 48 horas) em sistema fechado de recirculação de água. Os microrganismos analisados foram: coliformes termotolerantes, estafilococos coagulase positiva, contagem total de aeróbios mesófilos e Salmonella $s p$. Os resultados indicaram que houve uma diminuição dos microrganismos aeróbios mesófilos para < $10 \mathrm{UFC} / \mathrm{g}$ em 12 horas de depuração. No cultivo de Coruripe, verificou-se a presença de Salmonella sp, que foi eliminada das ostras em 24 horas de depuração. A depuração de ostra se mostrou eficiente para atender os padrões microbiológicos da resolução Anvisa - RDC $n^{\circ}$ 12/2001.
\end{abstract}

Palavras-chave: ostreicultura, microbiologia, contaminação.

\begin{abstract}
Oysters are bivalve and filter mollusks, which can trap pathogenic microorganisms in their tissues and cause public health issues to consumers in general. Thus, depuration is a process that aims to eliminate the pathogenic agents of bivalve mollusks, providing a product with full health assurance to the consumer market. This work had as main aim to achieve a microbiological evaluation of oysters grown on the coast of Alagoas and to compare the results with the microbiological standards demonstrated in Brazil, according to the Anvisa Resolution RDC No. 12 as of January 2, 2001. Oysters came from four different production sites, submitted to four periods of depuration (12, 24, 36 and 48 hours) in a closed water recirculation system. The analyzed microorganisms were: thermotolerant coliforms, coagulase positive staphylococci, total count of mesophilic aerobes, and Salmonella sp. The results indicated that there was a decrease of the aerobic mesophilic microorganisms to $<10 \mathrm{CFU} / \mathrm{g}$ in a 12-hour depuration. In the Coruripe cultivation, it was attested the presence of Salmonella sp, which was eliminated from the oysters in a 24- hour depuration period. The oyster depuration was shown as efficient to meet the microbiological standards of the Anvisa Resolution (12/2001).
\end{abstract}

Keywords: ostreiculture, microbiology, contamination. 


\section{Introdução}

O cultivo de moluscos bivalves no Brasil é uma atividade praticada em toda extensão da costa litorânea, sobretudo, no Estado de Santa Catarina que é responsável por mais de $90 \%$ da produção nacional, concentrada, majoritariamente, no mexilhão da espécie Perna perna e em ostras do pacifico, Crassostrea gigas (Epagri, 2016; FAO, 2016).

Dentre as espécies nativas, a ostra de mangue, Crassostrea brasiliana representa um importante recurso natural para as populações costeiras do Nordeste do Brasil e o Estado de Alagoas há mais de uma década se destaca no cultivo desse molusco exercido por pescadores e marisqueiras, que além de ser uma fonte de renda para as famílias, proporciona a inclusão social, manutenção dos estoques naturais e preservação dos manguezais (Nonô, 2010; Assad, Pimenta \& Sette, 2014).

Devido ao hábito alimentar filtrador das ostras, sua alimentação é baseada em partículas em suspenção na água, que pode acumular em seus tecidos microrganismos patogênicos presentes no ambiente de produção (Liang, He, Jiang, Chen, \& Yao, 2004; Newell, 2004; Pruzzo, Gallo \& Canesi, 2005), ocasionando intoxicação alimentar e problemas gastrintestinais aos consumidores (Leal \& Franco, 2008; Sande et al., 2010). Logo, o lançamento de esgotos em regiões próximas a produção, pode causar a poluição das águas do cultivo e consequentemente contaminar as ostras produzidas.

Diante disso, a depuração é um processo no qual os moluscos bivalves são colocados por um determinado tempo em água limpa, eliminando naturalmente, por meio da filtração da água, microrganismos presentes em seus tecidos (Lee, Lovatelli \& Ababouch, 2010; Rebouças \& Gonçalves, 2013). Esse processo é uma maneira eficiente de garantir a qualidade sanitária, além de proporcionar melhor qualidade sensorial dos moluscos bivalves cultivados.

No Brasil, a legislação que aborda os padrões microbiológicos para o pescado destinado ao consumo humano é regulamentada pela resolução da Anvisa RDC n⿳0 12, de 02 de janeiro de 2001, que exige, conforme item 7 "a", para o consumo de moluscos bivalves "in natura", unicamente as análises para Salmonella sp. e Staphylococcus coagulase positiva (Brasil, 2001).

Diante desse contexto, esse trabalho tem o objetivo de avaliar a depuração de ostras (Crassostrea brasiliana) provenientes de quatro locais de produção, submetidas a quatro tempos de depuração em sistema fechado de recirculação com tratamento da água por luz ultravioleta e filtros mecânicos.

\section{Material e Métodos}

O estudo foi realizado na unidade de depuração de moluscos, localizada no município de Coruripe/AL (10 9'35.92"S, 36 9'26.54"O), no mês de outubro de 2013. As amostras de ostras foram coletadas nos seguintes locais de cultivo existentes no Estado de Alagoas: Coruripe (povoado de Barreiras), Barra de São Miguel (povoado da Palatéia), Passo do Camaragibe (povoado de Barra do Camaragibe) e Porto de Pedras.

\section{COLETA E LIMPEZA DAS AMOSTRAS}

De cada local, foram coletadas, aleatoriamente, nas mesas de cultivo, 80 ostras adultas cultivadas em sistema de mesa fixa. Na unidade de depuração, as ostras foram lavadas e distribuídas em quatro caixas plásticas vazadas, pré-identificadas para cada local amostrado. Em seguida, as caixas foram colocadas nos tanques de depuração.

\section{SISTEMA DE DEPURAÇÃO}

O sistema de depuração foi composto por dois tanques de fibra $(4 \mathrm{~m} \times 1,5 \mathrm{~m} \times 1 \mathrm{~m})$ com capacidade de 4.600 L, dois filtros cartucho de $25 \mathrm{com}$ capacidade de $6 \mathrm{~m}^{3} / \mathrm{h}$, uma luz ultravioleta de $95 \mathrm{w}$, um filtro de areia com vazão de $6,5 \mathrm{~m}^{3} / \mathrm{h}$, uma caixa d' água de $1.000 \mathrm{~L}$ usada como tanque de passagem e uma bomba hidráulica de $1 \mathrm{CV}$ para recirculação da água (Figura 1). O sistema de recirculação tinha capacidade de operar a $12 \mathrm{~m}^{3} / \mathrm{h}$.

A água usada na depuração foi captada por meio de bombeamento diretamente do estuário e tratada com 3 ppm de cloro livre 12 horas antes de entrar no sistema.

\section{DEPURAÇÃO DAS OSTRAS}

Antes de iniciar a depuração, se retirou 10 ostras de cada localidade para análise no tempo zero hora (ostras sem depurar). As ostras restantes foram distribuídas em quatro caixas vazadas (uma para cada localidade) e submetidas a quatro tempos de depuração: 12, 24, 36 e 48 horas, coletando-se, aleatoriamente, 10 ostras a cada tempo para as análises microbiológicas. 


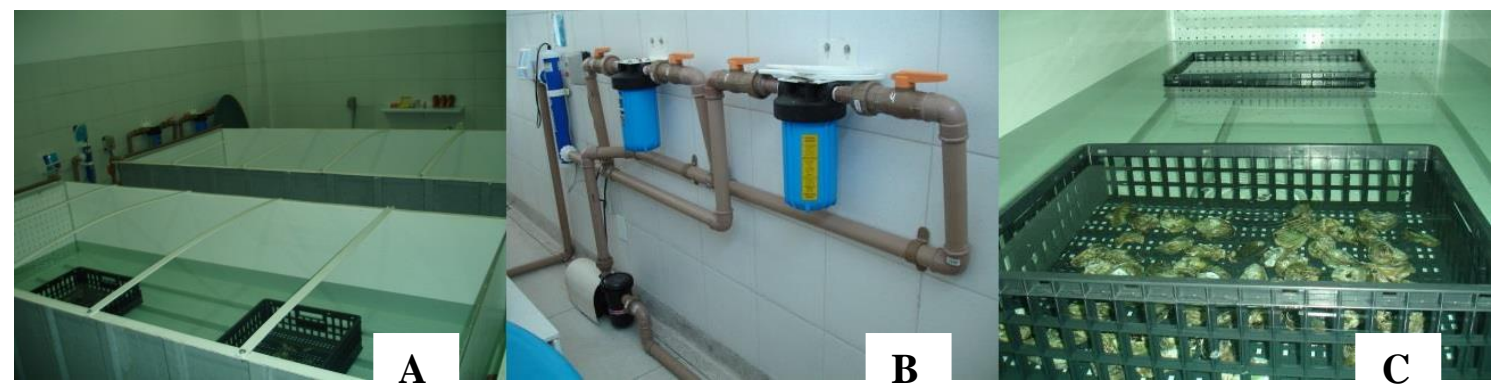

Figura 1. Sistema de depuração de ostras (A). Filtragem da água (B). Ostras em depuração (C).

A cada coleta de amostras, aferiu-se os seguintes parâmetros físico-químicos de qualidade da água: temperatura $\left({ }^{\circ} \mathrm{C}\right)$, salinidade $(\%), \mathrm{pH}$, condutividade elétrica $(\mu \mathrm{S} / \mathrm{cm})$ e TDS - sólidos dissolvidos totais (g/L), utilizou-se um medidor multiparâmetro portátil Hanna HI 9828.

\section{ANÁLISES MICROBIOLÓGICAS}

As análises microbiológicas foram realizadas no laboratório de microbiologia do curso de nutrição da Universidade Federal de Alagoas $\left(9^{\circ} 33^{\prime} 23.62^{\prime \prime} \mathrm{S}, 35^{\circ} 46^{\prime} 32.35^{\prime \prime O}\right)$, segundo metodologia descrita por Silva et al., (2010).

Os microrganismos analisados foram: Coliformes termotolerantes a $45^{\circ} \mathrm{C} / \mathrm{g}$, Estafilococos coagulase positiva/g, contagem total de aeróbios mesófilos (UFC/g) e Salmonella $s p / 25 \mathrm{~g}$. Os valores obtidos foram comparados com os padrões oficiais descritos na resolução da Anvisa - RDC no 12/2001 conforme item 7 "a", para o consumo de moluscos bivalves consumidos "in natura" (Brasil, 2001).

\section{Resultados e Discussão}

Durante a depuração observou-se que as ostras estavam com as valvas abertas realizando a filtração da água, indicando adaptação dos moluscos no ambiente de depuração. No decurso do estudo, não se observou mortalidade das ostras.

Os resultados das análises microbiológicas das ostras cultivadas em diferentes localidades no Estado de Alagoas são apresentados na Tabela 1.

Tabela 1. Análises microbiológicas das ostras cultivadas em quatro locais no Estado de Alagoas, submetidas a quatro tempos de depuração.

\begin{tabular}{|c|c|c|c|c|c|c|}
\hline \multirow{2}{*}{ Local } & \multirow{2}{*}{ Microrganismo } & \multicolumn{5}{|c|}{ Tempo de depuração } \\
\hline & & 0 hora & 12 horas & 24 horas & 36 horas & 48 horas \\
\hline \multirow{4}{*}{ Coruripe } & Coliformes termotolerantes (NMP) & 9 & 0 & 0 & 0 & 0 \\
\hline & Estafilococos coagulase positivo (UFC/g) & 0 & 0 & 0 & 0 & 0 \\
\hline & Contagem total de aeróbios mesófilos (UFC/g) & $<10$ & $10,3 \times 10$ & $<10$ & $<10$ & $<10$ \\
\hline & Salmonella $s p$ & Presença & Presença & Ausência & Ausência & Ausência \\
\hline \multirow{4}{*}{$\begin{array}{l}\text { Barra de São } \\
\text { Miguel }\end{array}$} & Coliformes termotolerantes (NMP) & 23 & 0 & 0 & 0 & 0 \\
\hline & Estafilococos coagulase positivo (UFC/g) & 0 & 0 & 0 & 0 & 0 \\
\hline & Contagem total de aeróbios mesófilos (UFC/g) & $1,8 \times 10$ & $1,1 \times 10$ & $<10$ & $<10$ & $<10$ \\
\hline & Salmonella sp & Ausência & Ausência & Ausência & Ausência & Ausência \\
\hline \multirow{4}{*}{$\begin{array}{l}\text { Passo do } \\
\text { Camaragibe }\end{array}$} & Coliformes termotolerantes (NMP) & 0 & 0 & 0 & 0 & 0 \\
\hline & Estafilococos coagulase positivo (UFC/g) & 0 & 0 & 0 & 0 & 0 \\
\hline & Contagem total de aeróbios mesófilos (UFC/g) & $<10$ & $1,6 \times 10$ & $<10$ & $<10$ & $<10$ \\
\hline & Salmonella $s p$ & Ausência & Ausência & Ausência & Ausência & Ausência \\
\hline \multirow{4}{*}{ Porto de Pedras } & Coliformes termotolerantes (NMP) & 0 & 0 & 0 & 0 & 0 \\
\hline & Estafilococos coagulase positivo (UFC/g) & 0 & 0 & 0 & 0 & 0 \\
\hline & Contagem total de aeróbios mesófilos (UFC/g) & $<10$ & $1,2 \times 10$ & $<10$ & $<10$ & $<10$ \\
\hline & Salmonella sp & Ausência & Ausência & Ausência & Ausência & Ausência \\
\hline
\end{tabular}

$\mathrm{NMP} / \mathrm{g}=$ Unidades formadoras de colônias por grama. 
Em todas as localidades, verificou-se que antes da depuração (0 hora) os valores obtidos para os coliformes termotolerantes e Estafilococos coagulase positivo foram menores que os limites estabelecido pela resolução da Anvisa - RDC no 12/2001, que devem ser de até $10^{2}$ (molusco refrigerado) e $10^{3} \mathrm{UFC} / \mathrm{g}$, respectivamente, para cada microrganismo (Brasil, 2001), devido a isso, nesse estudo, não foi possível avaliar a eficiência da depuração na eliminação desses microrganismos. Santos et al. (2016) verificou que no intervalo de 6 a 24 horas de depuração não houve diminuição significativa de coliformes termotolerantes em ostras Crassostrea rhizophorae, no entanto, a eficiência na eliminação desses microrganismos pela depuração é evidenciada em outros estudos (Martinez, Rodríguez-Calleja, Santos, Otero \& Garcia-Lopez, 2009; Love, Lovelace \& Sobsey, 2010; Obodai, Nyarko \& Amponsah, 2010).

Os valores obtidos para a contagem total de aeróbios mesófilos diminuíram para $<10 \mathrm{UFC} / \mathrm{g}$ em todos os locais amostrados a partir de 24 horas de depuração, este resultado corrobora com observado por Corrêa (2006) em ostras do pacifico C. Gigas que mostrou total eliminação de bactérias em 12 horas de depuração.

A resolução da Anvisa - RDC n⿳0 12/2001 não estabelece limites para a contagem total de aeróbios mesófilos em moluscos consumidos "in natura" (Brasil, 2001), no entanto, é um indicador importante de contaminação dos moluscos bivalves, sobretudo, aos que são consumidos "in natura".

No presente estudo, constatou-se a presença de Salmonella sp. nas amostras de ostras de Coruripe, porém, a partir de 24 horas de depuração, o sistema foi capaz de eliminar totalmente o microrganismo, o qual, de acordo a RDC $\mathrm{n}^{-}$12/2001 deve estar ausente (em $25 \mathrm{~g}$ de amostra) para o consumo de ostras "in natura" (Brasil, 2001). Essa patogenicidade encontrada está possivelmente associada à contaminação por esgoto doméstico não tratado despejados no Rio Coruripe, local onde as ostras são cultivadas, podendo causar problemas de saúde pública por meio da ingestão de moluscos contaminados (Barreto, de Sousa \& Fernandes Vieira, 2008; Melo, 2014; Silva, 2016). Esse estudo corrobora com Corrêa et al. (2007) que eliminou Salmonella Typhimurium em ostras C. Gigas depuradas durante 12 horas com água tratada por ultravioleta e cloro.

Os parâmetros físico-químicos de qualidade da água (temperatura, salinidade, $\mathrm{pH}$, condutividade elétrica e sólidos dissolvidos) se mantiveram estáveis durante as 48 horas de depuração (Tabela 2), devido a isso, evitou-se mortalidades e desovas das ostras que poderiam ocorrer com variações bruscas de temperatura e salinidade da água (Galvão, Pereira, Machado \& Henrique, 2000; Lavander et al., 2008).

A estabilidade dos parâmetros de qualidade da água durante a depuração é fundamental para manter os moluscos bivalves em equilíbrio osmorregulatório (Lima et al., 2009; Oliveira, Cunha, Castilho, Romalde \& Pereira, 2011) e assim obter a capacidade máxima de filtração dos organismos, proporcionando uma eliminação microbiana mais eficiente.

Os valores de TDS (sólidos dissolvidos totais) demonstraram que houve um aumento de $0,26 \mathrm{~g} / \mathrm{L}$ de partículas solidas entre 0 e 24 horas de depuração, possivelmente causado pela liberação de fezes e pseudofezes das ostras, não aumentando ao longo da depuração devido a retenção das partículas em suspensão nos filtros mecânicos do sistema de depuração.

Tabela 2. Parâmetros físico-químicos de qualidade da água durante 48 horas de depuração.

\begin{tabular}{lrrrrr}
\hline \multirow{2}{*}{ Parâmetros } & \multicolumn{7}{c}{ Tempos de depuração } \\
\cline { 2 - 6 } & 0 hora & 12 horas & 24 horas & 36 horas & 48 horas \\
\hline Temperatura $\left({ }^{\circ} \mathrm{C}\right)$ & 28,26 & 28,00 & 27,99 & 27,69 & 27,77 \\
Salinidade $(\% 0)$ & 37,40 & 37,41 & 37,83 & 37,78 & 37,67 \\
$\mathrm{pH}$ & 7,98 & 8,22 & 8,2 & 8,14 & 8,25 \\
Condutividade $(\mu \mathrm{S} / \mathrm{cm})$ & 56,42 & 56,41 & 56,94 & 56,89 & 56,76 \\
TDS - sólidos dissolvidos & 28,21 & 28,32 & 28,47 & 28,45 & 28,38 \\
totais $(\mathrm{g} / \mathrm{L})$ & & & & &
\end{tabular}

Os resultados deste estudo demostram que a depuração é uma tecnologia que deve ser aplicada nos moluscos bivalves cultivados visando fornecer um produto isento de contaminações, sobretudo, em acordo com a resolução da Anvisa - RDC n⿳ํㅡㄹ 12/2001. 


\section{Conclusões}

A depuração de ostras durante 12 horas diminuiu os microrganismos aeróbios mesófilos para < 10 UFC/g e em 24 horas eliminou o microrganismo Salmonella sp presente no tecido das ostras.

O processo de depuração se mostrou eficiente para atender os padrões microbiológicos da resolução Anvisa - $\mathrm{RDC} \mathrm{n}^{\mathrm{o}}$ 12/2001, fornecendo um produto com garantia sanitária aos consumidores.

\section{Agradecimentos}

Ao Instituto Brasileiro de Sustentabilidade pelo apoio a realização desse estudo.

\section{Referências}

Assad, L.T., Pimenta, M.C., \& Sette, I.R. (2014). A inserção de produtos de base comunitária na cadeia do Turismo: Um relato da experiência do projeto Ostras Depuradas de Alagoas - Brasil. In: UNITWIN UNESCO International Congress: Tourism and Gastronomy Heritage. Barcelona.

Barreto, N. E., de Sousa, O. V., \& dos Fernandes Vieira, R. H. S. (2008). Moluscos bivalves: Organismos Bioindicadores da Qualidade Microbiológica das Águas: Uma Revisão. Revista Brasileira de Higiene e Sanidade Animal, 2(2), 18-31.

Brasil (2001). Agência Nacional de Vigilância Sanitária. Resolução RDC n 12, de 2 de janeiro de 2001. Diário Oficial República Federativa do Brasil. Poder Executivo, Brasília, DF. Seção 1.

Corrêa, A. A. (2006). Estudo sobre a dinâmica de depuração de ostras de cultivo (Crassostrea gigas) artificialmente contaminadas com Salmonella enterica sorovar Typhimurium [Dissertação de Mestrado]. Florianópolis (SC): Universidade Federal de Santa Catarina.

Corrêa, A. A., Albarnaz, J. D., Moresco, V., Poli, C. R., Teixeira, A. L., Oliveira Simões, C. M. \& Monte Barardi, C. R. (2007). Depuration dynamics of oysters (Crassostrea gigas) artificially contaminated by Salmonella enterica serovar Typhimurium. Mar. Environ. Res., 63(5): 479-489. Doi 10.1016/j.marenvres.2006.12.002

Denton, G. R. W., \& Burdon-Jones, C. (1981). Influence of temperature and salinity on the uptake, distribution and depuration of mercury, cadmium and lead by the black-lip oyster Saccostrea echinata. Marine biology, 64(3): 317-326. Doi 10.1007/BF00393633

Empresa de Pesquisa Agropecuária e Extensão Rural de Santa Catarina (EPAGRI). (2016). Síntese Informativa da Maricultura 2015. Florianópolis. Acessado em 01 de fevereiro de 2017 em http://www.epagri.sc.gov.br/wp-content/uploads/2013/08/Sintese-informativa-da-maricultura-2015.pdf.

Food and Agriculture Organization of the United Nations (FAO). (2016). The State of World Fisheries and Aquaculture, SOFIA, Fisheries and Aquaculture Department. Roma. Acessado em 01 de fevereiro de 2017 em http://www.fao.org/fishery/sofia/en

Galvão, M. S. N., Pereira, O. M., Machado, I. C., \& Henrique, M. B. (2000). Aspectos reprodutivos da ostra Crassostrea brasiliana de manguezais do estuário de Cananéia, SP (25 S; $48 \mathrm{~W})$. Boletim do Instituto de Pesca, 26(2): 147-162.

Lavander, H. D., Junior, L. D. O. C., Oliveira, R. L. M., Santos, L. B. G., Neto, S. R. S., Souza, A. B., \& Gálvez, A. O. (2008). Diferentes métodos de indução a reprodução da ostra nativa Crassostrea rhizophorae (Guilding, 1828) em laboratório. Revista Brasileira de Engenharia de Pesca, 33, 12-14.

Leal, D. A. G. \& Franco, R. M. B. (2008). Moluscos bivalves destinados ao consumo humano como vetores de protozoários patogênicos: metodologias de detecção e normas de controle. Revista Panamericana de Infectologia, 10(4): 48-57.

Lee, R., Lovatelli, A. \& Ababouch, L. (2010). Depuracion de bivalvos: aspectos fundamentales y prácticos. FAO - Documento Técnico de Pesca 511. Roma, 153p.

Liang, L. N., He, B., Jiang, G. B., Chen, D. Y., \& Yao, Z. W. (2004). Evaluation of mollusks as biomonitors to investigate heavy metal contaminations along the Chinese Bohai Sea. Science of the Total Environment, 324(1): 105-113. Doi 10.1016/j.scitotenv.2003.10.021

Lima, M. D. A., Soares, M. D. O., Paiva, C. C., Osório, F. M., Porfírio, A. F., \& Cascon, H. M. (2009). Osmorregulação em moluscos: o caso do bivalve estuarino tropical Anomalocardia brasiliana (Mollusca: Bivalvia). Conexões-Ciência e Tecnologia, 3(1): 79-84.

Love, D. C., Lovelace, G. L., \& Sobsey, M. D. (2010). Removal of Escherichia coli, Enterococcus fecalis, coliphage MS2, poliovirus, and hepatitis A virus from oysters (Crassostrea virginica) and hard shell clams 
(Mercinaria mercinaria) by depuration. International journal of food microbiology, 143(3): 211-217. Doi 10.1016/j.ijfoodmicro.2010.08.028

Martinez, O., Rodríguez-Calleja, J. M., Santos, J. A., Otero, A., \& Garcia-Lopez, M. L. (2009). Foodborne and indicator bacteria in farmed molluscan shellfish before and after depuration. Journal of food protection, 72(7): 1443-1449. Doi 10.4315/0362-028X-72.7.1443

Melo, F. P. (2014). Crescimento urbano do município do Igaci-AL e perda da qualidade de vida. Periódico Técnico e Científico Cidades Verdes, 2(3).

Newell, R. I. (2004). Ecosystem influences of natural and cultivated populations of suspension-feeding bivalve molluscs: a review. Journal of Shellfish Research, 23(1): 51-62.

Nonô, R.G.B. (2010). Cultivo de Ostras em Alagoas. Maceió, SEBRAE/AL.

Obodai, E. A., Nyarko, H. D., \& Amponsah, S. L. (2010). Effect of depuration on microbial content of mangrove oyster (Crassostrea Tulipa) From Benya Lagoon, Ghana. Ethiopian Journal of Environmental Studies and Management, 3(2). Doi 10.4314/ejesm.v3i2.59832

Oliveira, J., Cunha, A., Castilho, F., Romalde, J. L., \& Pereira, M. J. (2011). Microbial contamination and purification of bivalve shellfish: Crucial aspects in monitoring and future perspectives-A minireview. Food Control, 22(6): 805-816. Doi 10.1016/j.foodcont.2010.11.032

Pruzzo, C., Gallo, G., \& Canesi, L. (2005). Persistence of vibrios in marine bivalves: the role of interactions with haemolymph components. Environmental Microbiology , 7 (6): p. 761-772. Doi 10.1111/j.1462-2920.2005.00792.x

Rebouças, L. D. O. S., \& Gonçalves, A. A. (2014). Uso de ozônio em sistemas de depuração de ostras visando a segurança para o consumidor. Acta of Fisheries and Aquatic Resources, 1(1): 49-90.

Sande, D., Melo, T. A., Oliveira, G. S. A., Barreto, L., Talbot, T., Boehs, G., \& Andrioli, J. L. (2010). Prospecção de moluscos bivalves no estudo da poluição dos rios Cachoeira e Santana em Ilhéus, Bahia, Brasil. Brazilian Journal of Veterinary Research and Animal Science, 47(3): 190-196. Doi 10.11606

Santos, S. S., Barreto, L. M., da Silveira, C. S., Reis, N. A., Lima, K. A., de Souza, J. D. S., \& EvangelistaBarreto, N. S. (2016). Condições sanitárias de ostras produzidas e comercializadas em Taperoá, Bahia e o efeito da depuração na redução da carga microbiana. Acta of Fisheries and Aquatic Resources, 3(2): 4960. Doi 10.2312

Silva, L. C. S. (2016). Importância das nascentes do Semiárido Alagoano no abastecimento das populações rurais difusas. Revista de Geociências do Nordeste, 2: 534-544.

Silva, N., Junqueira, V. C. A., Silveira, N. F. A., Taniwaki, M. H., Santos, R. F. S. \& Gomes, R. A. R. (2010). Manual de métodos de análise microbiológica de alimentos e água. Rio de Janeiro: Varela. 